DOI: https://doi.org/10.46296/yc.v4i6edesp.0025

\title{
PROCESOS ADMINISTRATIVOS Y FINANCIEROS PARA LA PROVISIÓN DE LOS FONDOS DE JUBILACIÓN PATRONAL DE LA UNIVERSIDAD LAICA ELOY ALFARO DE MANABÍ
}

\section{ADMINISTRATIVE AND FINANCIAL PROCESSES FOR THE PROVISION OF THE EMPLOYMENT RETIREMENT FUNDS OF THE UNIVERSIDAD LAICA ELOY ALFARO DE MANABÍ}

\author{
Barahona-Holguín Miguel Nemesio ${ }^{1 *}$; Bello-Sabando Borisse ${ }^{2}$ \\ ${ }^{1}$ Estudiante de la Maestría de Administración de Empresas/Instituto de \\ Posgrado/Universidad Técnica de Manabí. Portoviejo, Ecuador. \\ ${ }^{2}$ Magíster en Administración de Empresas. Profesor de la Facultad de Ciencias \\ Administrativas y Económicas, Universidad Técnica de Manabí. Portoviejo, Ecuador. \\ *Correo: barahonamiguel1@hotmail.com
}

\begin{abstract}
Resumen
La jubilación patronal es un derecho de los trabajadores y una obligación de los empleadores, se encuentra normalizada en el Código de Trabajo en los artículos 216, 217, 218 y 219. En este sentido, las empresas e instituciones públicas y privadas deben garantizar un adecuado proceso de provisión de los fondos de jubilación. La Universidad Laica Eloy Alfaro de Manabí es un centro de educación superior, que como institución de carácter público prioriza los derechos de sus trabajadores y por lo tanto, desde esta IES se gestionan adecuadamente los recursos de los trabajadores sin afectar la disponibilidad financiera y los procesos administrativos sujetos a las normativas legales vigentes. Por otra parte, los informantes que participaron en la investigación mencionan que conocen el caso de varios ex trabajadores de la ULEAM que se jubilaron en la institución y han recibido su jubilación patronal a partir de los fondos destinados para estos fines. Además, en la revisión documental se demuestra que la Ley para la justicia laboral incide en las finanzas de las instituciones de educación de tercer nivel, debido a que con la nueva fórmula, el valor aportado por el empleador es mayor y el tiempo de servicio del trabajador disminuye.
\end{abstract}

Palabras clave: Administración, jubilación, seguridad social, finanzas, Ley.

\begin{abstract}
Employer retirement is a right of workers and an obligation of employers, is standardized in the Labor Code in articles 216, 217, 218 and 219. In this sense, public and private companies must ensure adequate process of provision of retirement funds. The Universidad Laica Eloy Alfaro de Manabí is a center of higher education, which as a public institution prioritizes the rights of its workers and therefore, from this IES the resources of the workers are properly managed without affecting the financial availability and processes administrative subject to current legal regulations. On the other hand, the informants who participated in the investigation mention that they are aware of the case of several former ULEAM workers who retired at the institution and have received their employer's retirement from the funds allocated for these purposes. In addition, the documentary review shows that the Law for Labor Justice affects the finances of third level institutions, because with the new formula the value contributed by the employer is greater and the worker's service time decreases.
\end{abstract}

Keywords: Administration, retirement, social security, finance, Law.

Información del manuscrito:

Fecha de recepción: 13 de abril de 2020

Fecha de aceptación: 04 de mayo de 2020

Fecha de publicación: 05 de mayo de 2020 


\section{Introducción}

La Universidad Laica Eloy Alfaro de Manabí es una institución de educación superior (IES) ubicada en la ciudad de Manta, Manabí, Ecuador, que cuenta con extensiones en diferentes cantones de la provincia de Manabí. Esta IES fue fundada el 13 de noviembre de 1985 mediante la Ley 10 No. 10 Registro Oficial. 313, ha entregado más de 20.000 egresados y cuenta a la actualidad con 2212 trabajadores según el reporte del Departamento de Talento Humano de la IES (ULEAM, 2019). En el Ecuador la jubilación patronal es una obligación de los empleadores, normalizada en el Código del Trabajo reformado según los artículos 216, 217, 218 y 219. La pensión jubilar se basa en las normas establecidas por el Instituto Ecuatoriano de Seguridad Social, de modo que el haber individual del jubilado se determina mediante los siguientes parámetros: fondo de reserva del empleado, suma equivalente al $5 \%$ del promedio de la remuneración anual percibida en los 5 años anteriores multiplicada por los años de aportación. Según el artículo 218 del Código de Trabajo, la pensión jubilar resulta del valor del haber individual de jubilación dividido para el coeficiente estipulado en la respectiva tabla.

La jubilación laboral se otorga cuando una persona natural que ejerce activamente sus actividades profesionales en una institución o empresa finaliza su período de labores por diversos factores, entre ellos la edad, afectaciones en la salud o motivos personales (Aguilera et al., 2015).

Por su parte, la pensión jubilar goza de las garantías expuestas a continuación: se exenta del pago del impuesto a la renta; constituyen préstamos privilegiados de clase primera; en caso de fallecimiento del beneficiario, los herederos tienen el derecho a recibir la pensión durante un año; los empleados gozan del derecho a la jubilación patronal, a pesar de gozar del derecho a la jubilación otorgada por el Instituto Ecuatoriano de Seguridad Social (IESS); el trabajador jubilado tiene derecho a la decimotercera y decimocuarta pensión jubilar.

Cabe indicar que, según la Ley Orgánica de Régimen Tributario Interno sobre la jubilación patronal y sus deducciones al Impuesto a la Renta, en el capítulo IV "Depuración 
Revista Científica Multidisciplinaria Arbitrada YACHASUN. Volumen 4, Número 6 (Edición especial) ISSN: 2697-3456 Procesos administrativos y financieros para la provisión de los fondos de jubilación patronal de la Universidad Laica Eloy Alfaro de Manabí

de los Ingresos, Sección 1era: De las Deducciones", las instituciones que realicen provisiones por jubilación patronal, tienen la facultad de utilizar estos valores para deducir sus declaraciones de impuesto a la renta (SRI, 2019).

La responsabilidad social corporativa de instituciones como la ULEAM, no culmina cuando el trabajador se jubila, puesto que, desde este momento, surge la necesidad de garantizar la continuidad del bienestar del exempleado, otorgándole una pensión justa que le permita desenvolverse en su nueva condición social, en calidad de jubilado.

Las normativas internacionales de información financiera (NIIF) proponen que las provisiones por pensiones de jubilación patronal serán otorgadas a los empleados que cumplan como mínimo 10 años de trabajo en la misma empresa, tomando en cuenta aspectos administrativos, financieros $y$ demográficos. También es conocido que un plan de beneficios post empleo puede ser de carácter formal e informal en dependencia de los acuerdos a los que lleguen empleado y empleador (Moragas,
2012). Por lo tanto, el objetivo de la presente investigación es evaluar el proceso para la provisión de los fondos de jubilación patronal de la Universidad Laica Eloy Alfaro de Manabí desde las aristas administrativas y financieras.

\section{Materiales y métodos}

\subsection{Método}

La investigación realizada se fundamenta en el método descriptivo y analítico, puesto que se analizaron las variables asociadas con la jubilación patronal, además, busca exponer y evaluar el proceso de provisión de fondos de jubilación patronal de la Universidad Laica Eloy Alfaro de Manabí con enfoque cualitativo, debido a que la información fue recolectada a partir de entrevistas. Los informantes que participaron en la investigación corresponden a trabajadores con relación de dependencia de la Universidad Laica Eloy Alfaro de Manabí (Uleam), Manta, Ecuador durante el período comprendido entre noviembre-diciembre del año 2019.

La entrevista fue sistematizada mediante un instrumento de investigación que cuenta con un 
diseño estructurado con un apartado introductorio donde se indica el objetivo de la investigación, además, se solicita el tiempo de trabajo en la institución, sexo y edad del colaborador. La entrevista está conformada por 5 preguntas realizadas mediante la estrategia semiestructurada (Bernard, 1995); Brage et al., 2003).

La recolección de la información se realizó en la jornada laboral de la ULEAM, durante los días laborables de los meses de noviembre y diciembre. El horario escogido oscila entre las 08:00 y las 15:30. El diseño de las entrevistas permitió obtener información para validar los objetivos planteados, los cuales sustentan el análisis administrativo y financiero del proceso de provisión de los fondos de jubilación patronal en la ULEAM.

\subsection{Muestra}

De una población de 2212 trabajadores reportados por la Universidad Laica Eloy Alfaro de Manabí, se tomó una muestra representativa. Esta muestra fue calculada mediante la fórmula que se expone a continuación (AguilarBarojas, 2005):

$$
\boldsymbol{n}=\frac{N * z^{2} * p * q}{e^{2}(N-1)+z^{2} * p * q}
$$

El intervalo de confianza fue del $95 \%$ para un porcentaje de error del $5 \%$.

Donde:

$\mathrm{n}$ : Muestra.

N: Población.

p: valor proporcional de la población que presenta la variable en estudio $(p=0,5)$.

q: valor proporcional de la población que no presenta la variable en estudio $(q=0,5)$.

z: El nivel de confianza refleja el valor de z crítico. Para el presente estudio, con un $95 \%$ de confianza, el valor de $z$ es 1,96 .

e: porcentaje de error en la muestra.

$$
\begin{gathered}
\boldsymbol{n}=\frac{2212 * 1,96^{2} * 0,5 * 0,5}{0,05^{2}(2212-1)+1,96^{2} * 0,5 * 0,5} \\
\boldsymbol{n}=\frac{2124,4048}{6,4879}=327,44 \approx 327
\end{gathered}
$$

La investigación de campo se realizó con la muestra determinada mediante la fórmula propuesta en Aguilar-Barojas (2005). Cabe indicar que el muestreo fue aleatorio en todas las unidades académicas y administrativas de la ULEAM, es decir, todos los trabajadores 
Revista Científica Multidisciplinaria Arbitrada YACHASUN. Volumen 4, Número 6 (Edición especial) ISSN: 2697-3456 Procesos administrativos y financieros para la provisión de los fondos de jubilación patronal de la Universidad Laica Eloy Alfaro de Manabí

contaron con las mismas Por su parte, la documentación que probabilidades de ser seleccionados en la muestra. De las 327 personas entrevistadas, 178 son de sexo femenino y 149 de sexo masculino. Los rangos de edad de los participantes fueron 26-58 años y 2953 años para las mujeres y hombres, respectivamente.

Finalmente, a partir de la investigación descriptiva de tipo cuantitativa, se generó la discusión y análisis de los resultados obtenidos, con la finalidad de determinar causas y consecuencias del comportamiento evaluado en el desarrollo de la investigación. Se contrastó la información con reportes investigativos y manuscritos académicos realizados en otras instituciones públicas y privadas y considerando la debida metodología y materiales empleados.

\section{Resultados y discusión}

El artículo 216 del Código de Trabajo determina que los trabajadores tienen derecho a la jubilación patronal, siempre y cuando cumplan con las siguientes condiciones: trabajar en calidad de empleado amparado al Código de Trabajo y tener al menos 25 años de servicio.

se debe presentar en las inmediaciones del Ministerio de Trabajo es la siguiente: solicitud de cálculo de la jubilación patronal y en caso de ser empleado público debe adjuntar un documento que certifique el período al que el trabajador estuvo sujeto al Código de Trabajo (Ministerio del Trabajo, 2019).

Según la investigación propuesta por Ramírez-Yepéz et al. (2019), existen empresas del sector privado que según los reportes de gerentes y administradores se aplican las normas contables, no obstante, auditorías aplicadas sobre los reportes de los contadores reflejan que estas empresas no cumplen con el proceso de jubilación patronal a los trabajadores. Por lo tanto, se determina que, en esta institución privada, aproximadamente entre 20 y 30 personas cumplen con los requisitos de provisión de jubilación patronal. También es válido señalar, que esta empresa se expone a potenciales sanciones por el incumplimiento de las disposiciones de los organismos de regulación (Silva, 1989).

Las instituciones consideran que el proceso de provisión de fondos de 
jubilación patronal no tiene repercusión en las finanzas, por consiguiente, pueden resolver esta situación y cancelar estos pagos en la medida en la que se vayan presentando, esto debido a la disponibilidad de dinero o liquidez con la que cuentan estas empresas. Sin embargo, las organizaciones que no poseen unas finanzas favorables, pueden ver afectados sus intereses por sanciones legales impuestas por los organismos estatales de regulación.

Por otra parte, los informantes clave que participaron en la investigación reportan que otros trabajadores ya jubilados han recibido su pensión en condiciones normales, tal como exige la Ley y el Ministerio de Trabajo. También mencionan que existe la necesidad que, desde los departamentos de talento humano, se informe, capacite y se brinde información referente a este tipo de procesos que son un derecho de los trabajadores acorde a los años de servicios que han brindado a la institución.

Además, un segmento de la población entrevistada (30\%) desconoce el procedimiento a seguir para acceder a la jubilación, indicando que el proceso "puede ser tedioso y complicado cuando el trabajador no tiene mucha cercanía con las autoridades o encargados del proceso".

Los trabajadores afiliados a la ULEAM manifiestan que confían en la gestión y administración de los fondos de jubilación, no obstante, surgen interrogantes relacionadas con la sostenibilidad a largo plazo y el efecto que tendrá a futuro en las finanzas de este centro de estudio.

Los efectos de la jubilación patronal sobre las finanzas de una institución deben ser tomados en cuenta, puesto que según Salazar (2018) el $67 \%$ de los trabajadores jubilados en las Universidades del Distrito Metropolitano de Quito, recibe una jubilación patronal de entre \$ 0-700. Por otra parte, la tercera parte de estas universidades no mantiene una provisión para el pago de la jubilación patronal, esto se asocia con que la mayoría de estas instituciones de educación superior pertenecen al sector público y por lo tanto sus finanzas dependen del Estado ecuatoriano. Por otra parte, las normativas internacionales de contabilidad como NIC 19, son aplicadas en la muestra evaluada en 
dicha investigación, de modo que aproximadamente el $90 \%$ de las universidades que realizan la provisión de los fondos de jubilación, emplean las normas contables mencionadas. La debida aplicación de las NIC 19 garantiza el cumplimiento de las exigencias de las leyes ecuatorianas en materia de jubilación patronal.

En la ley de justicia laboral se presentan reformas que buscan cambiar los tiempos de aportación y el período de prestación de servicios. Sin embargo, desde la perspectiva del empleador, esta ley afecta las finanzas de las instituciones tanto públicas y privadas. En este sentido, un $50 \%$ de las instituciones de educación superior, presentan un impacto directo en su economía debido a la aplicación de la ley para la justicia laboral, puesto que, mediante la fórmula de cálculo, aumenta el pago de pensión jubilar. Como sugerencia se plantea, modificar el número de imposiciones aportadas por el afiliado (Salazar, 2018).

\section{Conclusiones}

Se concluye que la provisión de fondos de jubilación patronal es una obligación de carácter legal, no obstante, un gran número de empresas incumple este requisito en función de sus políticas administrativas y financieras.

Es también notable que, a pesar de conocer los requisitos legales en materia de jubilación patronal, la alta dirección considera poco influyente y de resolución inmediata cuando se presentan. Por tanto, desde las instituciones estatales de regulación, debe existir mayor rigurosidad y obligatoriedad en el cumplimiento de los tiempos y protocolos que exige la ley, para de esta forma garantizar que el jubilado reciba la compensación por el tiempo y los servicios brindados a la institución.

La Universidad Laica Eloy Alfaro de Manabí es un centro de educación superior, que como institución de carácter público prioriza los derechos de sus trabajadores y por lo tanto, desde esta IES se gestionan adecuadamente los recursos de los trabajadores sin afectar la disponibilidad financiera y los procesos administrativos sujetos a las normativas legales.

\section{Bibliografía}

Aguilar-Barojas, S. (2005). Fórmulas para el cálculo de la muestra en 
investigaciones de salud. Salud en Tabasco, 11(1-2), 333-338.

Aguilera, M., Acosta, M., \& Pozos, B. (2015). 101 consejos para una jubilación satisfactoria: Aportaciones científicas y su aplicación práctica. México: Universitaria.

Bernard, H. (1995). Entrevistas no estructuradas $y$ semiestructuradas. Métodos de investigación en antropología, 167-179.

Brage, L. B., Socias, M., \& Torelló, J. (2003). Análisis cualitativo de entrevistas. Nómadas (18), 140-149.

Ministerio de Trabajo. (2019). Jubilación Patronal. Quito, Ecuador. Disponible en: http://www.trabajo.gob.ec/ju bilacion-patronal/

Moragas, R. (2012). Jubilación Siglo XXI: Salud. España: Copyright.

Ramírez-Yepéz, P., Ortiz-Segura, G., \& Tacuri-Coello, A. (2019). Provisiones para jubilación patronal y desahucio y su incidencia en los estados financieros. Revista Observatorio de la Economía Latinoamericana. Disponible en línea: https://www.eumed.net/rev/o el/2019/01/provisionesjubilacion-patronal.html

Salazar, C. (2018). Análisis del efecto causado por la aplicación de la Ley para la justicia laboral (pago de pensiones de los jubilados del Instituto Ecuatoriano de Seguridad Social), en las universidades del D.M.Q. Trabajo de titulación previo a la obtención del título de: Ingeniera en contabilidad y auditoría. Quito, Ecuador. Universidad Politécnica Salesiana.

Silva, M. (1989). Estudio sobre la jubilación patronal en el Ecuador. Quito: Gráficas San Pablo.

SRI. (2019). Ley Orgánica de Régimen Tributario Interno. Sistema de Rentas Internas. Quito, Ecuador. Obtenido: https://www.sri.gob.ec/Docu mentosAlfrescoPortlet/desc argar/f127300b27b5-4a80- 


\section{a27983fbf3aac667/LEY_DE}

\section{_RGIMEN_TRIBUTARIO_IN}

TERNO

ULEAM. (2019). Universidad Laica "Eloy Alfaro" de Manabí ULEAM. Historia. Disponible en:

https://www.uleam.edu.ec/hi storia/ 